\title{
Echocardiographic comparison of regional wall motion abnormality between patients with acute anteroseptal and acute extensive anteior ST segment elevation myocardial infarction
}

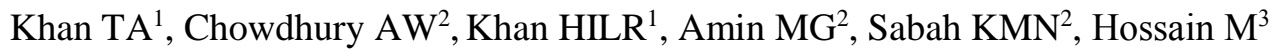 \\ ${ }^{1}$ Department of Cardiology, Anwer Khan Modern Medical College \& Hospital, Dhaka, ${ }^{2}$ Department of \\ Cardiology, Dhaka Medical College, Dhaka, ${ }^{3}$ Upazila Health Complex, Basail, Tangail. \\ Email:tunaggina.khan@gmail.com
}

\begin{abstract}
Post myocardial infarction (MI) short and long term clinical outcome is largely determined by the size of the infarcted area. It is generally assumed that as the lead involvement in electrocardiography (ECG) is less in anteroseptal ST segment elevation myocardial infarction (AS-STEMI), where ST segment elevation (STE) is limited to leads $\mathrm{V}_{1}$ to $\mathrm{V}_{3}$, myocardial damage is likely to be less; and in extensive anterior STEMI (EA-STEMI), as the STE extends further upto V6, the myocardial damage is likely to be more. This study was intended to compare regional wall motion abnormality (RWMA) between acute anteroseptal STEMI and acute extensive anterior STEMI patients. 90 patients with AS-STEMI and 106 patients with EA-STEMI, admitted in between October 2012 and September 2013, were included. For each patient, a transthoracic echocardiogram (TTE) was performed within 24-48 hours of MI and was interpreted by an independent investigator blinded to the patient's ECG data. No differences were observed between the two groups in baseline characteristics; except AS-STEMI group had more patients with diabetes and EA-STEMI group had more patients with family history of coronary artery disease. Distribution, extent of wall motion abnormalities and mean number of total involved segments were similar between patients with AS-STEMI and those with EA-STEMI ( $\mathrm{p}>0.05)$. Regarding regional dysfunction, the apical septal $(99.1 \%$ vs. $92.2 \%, \mathrm{p}<0.05)$ and apical $(76.4 \%$ vs. $60.0 \%, \mathrm{p}<0.05)$ segments were the only two segments that were affected significantly more in patients with EA-STEMI than in patients with AS-STEMI. So, the term AS-STEMI may be a misnomer, as it implies that only the anteroseptal segments of the left ventricle are involved. This study shows that regional dysfunction in patients with AS-STEMI extends beyond the anteroseptal region. So, any patients with anterior wall involvement, either anteroseptal or extensive anterior STEMI, should be treated with equal importance.
\end{abstract}

\section{Introduction}

Regional wall motion abnormality is one of the earliest features of acute MI even before the infarctive change is evident in standard ECG or by rise of cardiac biomarker. Echocardiography can detect RWMA and it is widely available, noninvasive and relatively cheap as well. ${ }^{1}$ STEMI is the most lethal form of acute MI (AMI). ${ }^{1}$

Anterior ST segment elevation myocardial infarction (A-STEMI) involves the territory of the myocardium supplied by the major artery of the heart i.e. left anterior descending artery (LAD). It can be commonly classified as anteroseptal STEMI and extensive anterior STEMI. ${ }^{2-4}$

Conventionally, ECG leads are said to be oriented according to the anatomic zones of the left ventricle i.e. $\mathrm{V}_{1}-\mathrm{V}_{3}$ for anteroseptal zone, $\mathrm{V}_{4}-\mathrm{V}_{6}$ for apical or lateral zone, I and aVL for high lateral zone. However, these conventional electrodes cannot be pinpointed or placed directly upon the heart itself and are situated some distance away; therefore a large area of myocardial injury may be substantially attenuated on surface ECG. ${ }^{3}$

Shalev et al. ${ }^{5}$ suggested that, only the anteriorseptum is never exclusively affected in patients with AS-STEMI. Porter et al. ${ }^{6}$ showed that STE in lead $V_{1}$ and lead $V_{2}$ is associated with different areas of infarction rather than septum. Bogaty et al. ${ }^{7}$ suggested that AS-STEMI is associated with an area of infarction predominantly involving the apex. But the apex may be involved either in anteroseptal, extensive anterior, or left dominant system occlusion related inferolateral MI. Bandeali et al. ${ }^{4}$ showed that AS-STEMI causes similar area of left ventricular involvement as that in EA- 
STEMI. Aldrich et al. ${ }^{8}$ suggested that the number of leads with ST segment elevation correlates with the infarct size in patients with A-STEMI, implying that AS-STEMI is associated with an infarct size smaller than that in patients with EA-STEMI. However neither angiographic nor echocardiographic data has correlated with either injury pattern with the expected location of injury. ${ }^{5-7,9}$ Two explanations regarding this matter opposing each other have been given; i.e. ASSTEMI is associated with a relatively small area of infarction, or it may be a manifestation of a large area of infarction, caused by a proximal occlusion of a wrapping left anterior descending artery (LAD) where STE in inferolateral leads (leads $\mathrm{V}_{4}$ to $V_{6}$ ) is cancelled by an opposing injury vector involving the basal segments, i.e. the anterosuperior leads (leads $\mathrm{V}_{1}$ and $\mathrm{V}_{2}$ ). ${ }^{5,9-11}$

If MI was confined to the anterior septum, as it is thought, in so called AS-STEMI, it would require an isolated occlusion of the principal septal artery, and such an occurrence is unusual. An infarction of the anterior septum also would be expected to implicate occlusion of proximal LAD and should therefore be extensive. ${ }^{7}$ Zhong et al. ${ }^{10}$ found that STE in lead $V_{1}$ occurs when LAD is occluded proximal to first septal branch $\left(S_{1}\right)$, which indicates that large area of myocardium is jeopardized which is perfused distal to $S_{1}$ and moreover, no significant difference was observed regarding the occlusion site between AS-STEMI and EA-STEMI in relation to the $S_{1}$ or first diagonal branch $\left(D_{1}\right)$ of LAD.

Whether AS-STEMI is truly an infarction involving smaller area of the left ventricular myocardium or it is as extensive as EA-STEMI has not been well studied. The few studies carried out abroad were done with small sample size i.e. less than 100 patients. This study was intended to compare echocardiographic RWMA in acute ASSTEMI and acute EA-STEMI patients with larger sample size.

\section{Materials and Methods}

This cross sectional analytical study was carried out in the Department of Cardiology, Dhaka Medical College Hospital, over a period of one year, from October, 2012 to September, 2013. By purposive sampling technique, 196 patients were selected. Anterior STEMI was defined as diagnostic new STE at the $\mathrm{J}$ point in at least 2 contiguous leads of $2 \mathrm{~mm}(0.2 \mathrm{mV})$ in men or 1.5 $\mathrm{mm}(0.15 \mathrm{mV})$ in women in leads $\mathrm{V}_{2}-\mathrm{V}_{3}$ and/or of $1 \mathrm{~mm}(0.1 \mathrm{mV})$ in other contiguous chest leads or the limb leads, provided there was no evidence of left ventricular hypertrophy or left bundle-branch block. ${ }^{12}$ AS-STEMI was denoted if STEMI was confined to leads $\mathrm{V}_{1}-\mathrm{V}_{3}$ and EA-STEMI was diagnosed when STEMI was confined to leads $\mathrm{V}_{1^{-}}$ $\mathrm{V}_{6}, \pm \mathrm{I}, \mathrm{aVL} .2,3,12$

Patients with history of old myocardial infarction/ intracoronary intervention/ coronary artery bypass grafting, STE in ECG other than MI i.e. pericarditis, prinzmetal angina, Brugada syndrome etc., ECG evidence of LBBB, WPW syndrome, ventricular arrhythmia, advanced second degree or third degree conduction defect, ventricular electronic pacing of heart, patients with cardiomyopathy, valvular heart disease, severe comorbid conditions such as ESRD, cirrhosis of liver, malignancy etc., patients who were not thrombolysed, and patients with poor echocardiographic windows were all excluded.

ECG was done immediately after admission and was interpreted by an expert cardiologist. TTE was done between 24-48 hours of MI by an expert echocardiographer blinded to the ECG diagnosis. These patients were categorized into two groups. Those with STE confined to leads $\mathrm{V}_{1}-\mathrm{V}_{3}$ were denoted as group I (anteroseptal STEMI or ASSTEMI) and those with STE in leads $\mathrm{V}_{1}-\mathrm{V}_{6} \pm \mathrm{I}$, aVL were denoted as group II (extensive anterior or EASTEMI).

The left ventricle was divided into 17 segments (six basal, six mid-ventricular, five apical). ${ }^{13}$ RWMA by TTE was assessed from each study subject according to the 17 segment model of left ventricle (Fig.1) in parasternal long axis, parasternal short axis, apical two chamber and apical four chamber views. RWMA detected in at least one segment was considered as involved and the results were recorded as normal, hypokinetic, akinetic and dyskinetic wall motion. Comparison of RWMA between the two groups for each of the 17 segments was done. Global wall motion abnormality was compared between the groups on the basis of ejection fraction (EF\%).

Data were expressed as mean \pm SD for continuous variables and as numbers (percent) for categorical variables. Continuous variables were compared by the paired-samples student t-test. Proportions were compared by Chi-square statistics; Fisher's exact test was used where appropriate. The 95\% confidence intervals (CIs) were calculated for each technique. Differences were considered significant at the 0.05 level and the power of the study was set as $90 \%$. All statistical calculations were performed using SPSS for Windows (version 16). 


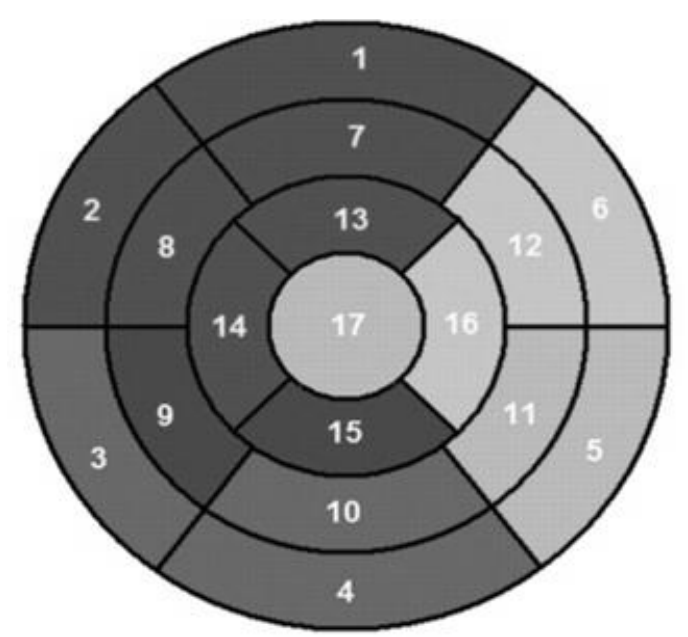

$\begin{array}{lll}\text { 1. Basal anterior } & \text { 7. Mid-anterior } & \text { 13. Apical anterior } \\ \text { 2. Basal anteroseptal } & \text { 8. Mid-anteroseptal } & \text { 14. Apical septal } \\ \text { 3. Basal inferoseptal } & \text { 9. Mid-inferoseptal } & \text { 15. Apical inferior } \\ \text { RCA } & \\ \text { 4. Basal inferior } & \text { 10. Mid-inferior } & \text { 16. Apical lateral } \\ \text { 5. Basal inferolateral } & \text { 11. Mid-inferolateral } & \text { 17. Apex } \\ \text { 6. Basal anterolateral } & \text { 12. Mid-anterolateral } & \end{array}$ LCX or LAD
LCX or RCA

Fig.1: 17 segment model of left ventricle with respective coronary circulation. ${ }^{13}$

\section{Results}

The age distribution of the study subjects was similar in both AS-STEMI and EA-STEMI groups [mean $( \pm \mathrm{SD})$ age $52.58( \pm 12.02)$ years vs. 50.72 $( \pm 13.22)$ years, $p>0.05]$. Gender distribution was similar in both AS-STEMI and EA-STEMI groups (91.1\% male and $8.9 \%$ female vs. $83 \%$ male and $17 \%$ female, $\mathrm{p}>0.05)$.

In group I, 54(60.0\%) patients and in group II, 36 (34.0\%) patients had history of diabetes mellitus. Group I had significantly more diabetic patients $(60 \%$ vs. $34 \%, \mathrm{p}<0.05)$. Group II had significantly more patients with family history of CAD $(31.1 \%$ vs. $47.2 \%, \mathrm{p}<0.05)$. No statistically significant difference was observed regarding obesity, hypertension, dyslipidaemia, sedentary life style, and smoking status between two groups.

More than $90 \%$ of the study subjects in both groups had predominant involvement of mid anterior, mid anteroseptal, mid inferoseptal, apical anterior, apical septal walls, and a significant number of study subjects had basal anterior, basal anteroseptal, apical anterior, apical septal segments involvement, indicating typical involvement of LAD territory. Variable degree of regional wall motion abnormality is seen in other segments indicating involvement of overlapping zones of LAD-RCA (right coronary artery) and LAD-LCX (left circumflex) artery supply.

In the present study, mean $( \pm \mathrm{SD})$ number of total involved segments in group I and group II patients were $8.83( \pm 2.49)$ and $9.01( \pm 2.25)$ respectively, with no statistically significant difference $(\mathrm{p}>0.05)$ (Table-I). When research assessed regional dysfunction, the apical septal or segment 14 (99.1\% vs. $92.2 \%, \mathrm{p}<0.05)$ and apical or segment $17(76.4 \%$ vs. $60.0 \%, \mathrm{p}<0.05)$ were the only two segments that were affected significantly more in patients with EA-STEMI than in patients with ASSTEMI (Table-II).

There was no significant difference in global wall motion abnormality as assessed by measuring mean $( \pm \mathrm{SD})$ ejection fraction $(\mathrm{EF} \%)$ between patients with AS-STEMI or EA-STEMI [38.80\% $( \pm 5.78)$ and $39.21 \%( \pm 5.90) ; p>0.05]$ (Table-III).

Table I: Comparison of mean of total involved segments between two groups $(\mathrm{n}=196)$

\begin{tabular}{|c|c|c|c|c|}
\hline \multirow{2}{*}{$\begin{array}{l}\text { Total } \\
\text { segments }\end{array}$} & \multirow[t]{2}{*}{ involved } & \multicolumn{2}{|c|}{ Groups } & \multirow[b]{2}{*}{$\mathrm{P}(95 \% \mathrm{CI})$} \\
\hline & & $\begin{array}{c}\text { Group I } \\
(\mathrm{n}=90)\end{array}$ & $\begin{array}{c}\text { Group II } \\
(\mathrm{n}=106)\end{array}$ & \\
\hline Mean \pm SD & & $8.83 \pm 2.49$ & $9.01 \pm 2.25$ & $\begin{array}{c}0.604^{\mathrm{NS}} \\
(.492 \text { to } .498)\end{array}$ \\
\hline Range & & $3-15$ & $4-15$ & \\
\hline
\end{tabular}

NS = not significant

Table II: Comparison of presence of left ventricular segmental wall motion abnormality (hypokinetic, akinetic and dyskinetic wall motion) with respective echocardiographic left ventricular segments between two groups $(n=196)$.

\begin{tabular}{llccc}
\hline \multicolumn{1}{c}{ LV segmental wall motion } & \multicolumn{2}{c}{ Groups } & \multirow{2}{*}{ p } \\
\cline { 3 - 4 } & & $\begin{array}{c}\text { Group I } \\
(\mathrm{n}=90)\end{array}$ & $\begin{array}{c}\text { Group II } \\
(\mathrm{n}=106)\end{array}$ & \\
\hline Segment 1 & Basal anterior & $38(42.2 \%)$ & $35(33.0 \%)$ & $0.184^{\mathrm{NS}}$ \\
Segment 2 & Basal anteroseptal & $42(46.7 \%)$ & $43(40.6 \%)$ & $0.390^{\mathrm{NS}}$ \\
Segment 3 & Basal inferoseptal & $34(37.8 \%)$ & $48(45.3 \%)$ & $0.288^{\mathrm{NS}}$ \\
Segment 4 & Basal inferior & $8(8.9 \%)$ & $6(5.7 \%)$ & $0.382^{\mathrm{NS}}$ \\
Segment 5 & Basal inferolateral & $2(2.2 \%)$ & $2(1.9 \%)$ & $0.869^{\mathrm{NS}}$ \\
Segment 6 & Basal anterolateral & $4(4.4 \%)$ & $1(0.9 \%)$ & $0.121^{\mathrm{NS}}$ \\
Segment 7 & Mid- anterior & $86(95.6 \%)$ & $100(94.3 \%)$ & $0.700^{\mathrm{NS}}$ \\
Segment 8 & Mid -anteroseptal & $88(97.8 \%)$ & $104(98.1 \%)$ & $0.869^{\mathrm{NS}}$ \\
Segment 9 & Mid -inferoseptal & $88(97.8 \%)$ & $100(94.3 \%)$ & $0.225^{\mathrm{NS}}$ \\
Segment 10 Mid- inferior & $16(17.8 \%)$ & $20(18.9 \%)$ & $0.844^{\mathrm{NS}}$ \\
Segment 11 Mid- inferolateral & $2(2.2 \%)$ & $6(5.7 \%)$ & $0.225^{\mathrm{NS}}$ \\
Segment 12 Mid-anterolateral & $56(62.2 \%)$ & $68(64.2 \%)$ & $0.780^{\mathrm{NS}}$ \\
Segment 13 Apical anterior & $88(97.8 \%)$ & $104(98.1 \%)$ & $0.869^{\mathrm{NS}}$ \\
Segment 14 Apical septal & $83(92.2 \%)$ & $105(99.1 \%)$ & $0.016^{\mathrm{S}}$ \\
Segment 15 Apical inferior & $34(37.8 \%)$ & $40(37.7 \%)$ & $0.995^{\mathrm{NS}}$ \\
Segment 16 Apical lateral & $72(80.0 \%)$ & $92(86.8 \%)$ & $0.200^{\mathrm{NS}}$ \\
Segment 17 Apical & $54(60.0 \%)$ & $81(76.4 \%)$ & $0.013^{\mathrm{S}}$ \\
\hline
\end{tabular}

NS = not significant, $\mathrm{S}=$ significant.

Table III: Comparison of mean of ejection fraction between two groups $(n=196)$

\begin{tabular}{lccc}
\hline Ejection fraction & \multicolumn{2}{c}{ Groups } & \\
\cline { 2 - 3 } & $\begin{array}{c}\text { Group I } \\
(\mathrm{n}=90)\end{array}$ & $\begin{array}{c}\text { Group II } \\
(\mathrm{n}=106)\end{array}$ & $\begin{array}{c}\mathrm{P} \\
(95 \% \mathrm{CI})\end{array}$ \\
\hline Mean \pm SD & $38.8 \pm 5.78$ & $39.21 \pm 5.90$ & $\begin{array}{c}0.627^{\mathrm{NS}} \\
(-2.059 \text { to } 1.245)\end{array}$ \\
\hline
\end{tabular}

$\mathrm{NS}=$ not significant, $\mathrm{S}=$ significant 


\section{Discussion}

Age distribution in both groups in our study was similar to Bandeali et al. ${ }^{4}$, Porter et al. ${ }^{6}$, Huang et al. ${ }^{9}$, Zafrir et al. ${ }^{14}$ and similarity in gender distribution was also found between our study and several other similar studies., ${ }^{4,6,9}$

Comparison of traditional risk factors revealed that AS-STEMI group had significantly more patients with diabetes mellitus and family history of CAD was significantly more prevalent in EA-STEMI patients. But, as a whole, no significant difference was observed regarding the prevalence of smoking, hypertension, dyslipidaemia, sedentary life style and obesity between the groups, as was observed by Huang et al. ${ }^{9}$

The results of our study showed that there was no significant difference in global wall motion abnormality as assessed by measuring ejection fraction between patients with AS-STEMI and EASTEMI. Our finding is consistent with Bandeali et al. ${ }^{4}$ who reported mean ( \pm SD) EF\% was $39.17 \%$ $( \pm 10.5)$ vs. $44.08 \%( \pm 10.5)$ with $p>0.05$. The same was found by some other researchers. ${ }^{6}$

In the present study, mean $( \pm S D)$ number of total involved segments in group I and group II patients were $8.83( \pm 2.49)$ and $9.01( \pm 2.25)$ respectively with no statistically significant difference. This finding was supported by Bandeali et al. ${ }^{4}$ where mean number of total involved segments were 6.5 $( \pm 4)$ and $8( \pm 4.5)$ in AS-STEMI and EA-STEMI groups respectively with no statistically significant difference.

When we assessed regional dysfunction, the apical septal or segment 14 and true apical or segment 17 were the only two segments that were affected significantly more in patients with EA-STEMI than in patients with AS-STEMI. In patients with EASTEMI, a trend was observed toward a greater degree of regional dysfunction in the basal inferoseptal or segment $3(45.3 \%$ vs. $37.8 \%$, $\mathrm{p}>0.05)$, mid inferolateral or segment $11(5.7 \%$ vs. $2.2 \%, \mathrm{p}>0.05)$, mid anterolateral or segment 12 $(64.2 \%$ vs. $62.2 \%, \mathrm{p}>0.05)$, apical lateral or segment $16(86.8 \%$ vs. $80.0 \%, p>0.05)$ but the differences were not statistically significant. On the other hand, in patients with AS-STEMI, a trend was observed toward a greater degree of regional dysfunction in the basal anterior or segment 1 $(42.2 \%$ vs $33.0 \%, \mathrm{p}>0.05)$, basal anteroseptal or segment $2(46.7 \%$ vs $40.6 \%, \mathrm{p}>0.05)$, basal inferior or segment $4(8.9 \%$ vs $5.7 \%, \mathrm{p}>0.05)$, basal anterolateral or segment $6(4.4 \%$ vs $0.9 \%, \mathrm{p}>0.05)$, mid inferoseptal or segment $9(97.8 \%$ vs $94.3 \%$, $\mathrm{p}>0.05)$, but the differences were not statistically significant. The incidence of the involvement of basal inferolateral or segment $5(1.9 \%$ vs $2.2 \%$, $\mathrm{p}>0.05)$, mid anterior or segment $7(94.3 \%$ vs $95.6 \%, \mathrm{p}>0.05)$, mid anteroseptal or segment 8 (98.1\% vs $97.8 \%, \mathrm{p}>0.05)$, mid inferior or segment $10(18.9 \%$ vs $17.8 \%, \mathrm{p}>0.05)$, apical anterior or segment $13(98.1 \%$ vs $97.8 \%, \mathrm{p}>0.05)$, apical inferior or segment $15(37.7 \%$ vs $37.8 \%$, p>0.05) were similar between patients with EA-STEMI and AS-STEMI.

More than $90 \%$ of the study subjects in both groups had predominant involvement of mid anterior, mid anteroseptal, mid inferoseptal, apical anterior, apical septal walls, and a significant number of study subjects had basal anterior, basal anteroseptal, apical anterior, apical septal segments involvement, indicating typical involvement of LAD territory. Variable degree of regional wall motion abnormality seen in other segments indicates involvement of overlapping zones of LAD-RCA and LAD-LCX artery supply.

Our study was in agreement with Bandeali et al. ${ }^{4}$ They studied 65 patients with anterior STEMI who underwent TTE within 24-48 hours of admission. They also showed a significant proportion of the subjects having involvement of mid anterior, mid anteroseptal, mid inferoseptal, apical anterior, apical septal, true apical segments and a moderate number of study subjects having involvement of basal anterior, basal anteroseptal segments. This finding was consistent with the current study with the exception of apical inferior segment (segment 15) where dysfunction occurred more often in patients with EA-STEMI than in patients with ASSTEMI $\quad(71.4 \%$ vs. $43.3 \% ; \mathrm{p}<0.05)$. The discrepancy can be explained by the presence of an alternative blood supply to the apical segments (because of the presence large diagonal or obtuse marginal branches or even branch of RCA), apical areas may be spared or involved in varying degree; moreover apex is an overlapping zone of LADRCA and LAD-LCX arteries. ${ }^{15}$

Huang et al. ${ }^{9}$ reported two contrasting angiographic findings and their relation to left ventricular segments of involvement among patients with ASTEMI. First, many patients with AS-STEMI than patients with EA-STEMI (52\% vs. 15\%) had proximal occlusion of a short LAD which did not reach the apex and an alternative blood supply to the apex by a large side branch, indicating more involvement of basal and mid segments sparing apical segments. In contrast, more patients with AS-STEMI than patients with EA-STEMI (52\% vs. $15 \%$ ) had a proximal occlusion of a long wraparound LAD, suggesting extensive left ventricular area of involvement. This is consistent with the current study because of the similarities in wide 
range of involvement of left ventricular segments, variable involvement of apical segments. Their angiographic findings are supported by the echocardiographic findings of our study, suggesting AS-STEMI is not always limited to the anteroseptal segment.

Moreover, Porter et al. ${ }^{6}$ showed comparable global left ventricular function and regional dysfunction irrespective of the presence of STE in lead $V_{5}$ or $\mathrm{V}_{6}$. No difference in regional dysfunction was observed between the patients with AS-STEMI and EA-STEMI showing similar involvement of basal anterior, basal anteroseptal, basal inferoseptal, and apical inferior segments between the groups without any statistical significance. Involvement of basal segments indicates proximal occlusion of LAD and extensive damage to left ventricle in both AS-STEMI and EA-STEMI. All these evidences make it clear that each electrode records the global activation vector toward and away from each lead and not the local events adjacent to the electrode. Therefore, leads $V_{1}$ to $V_{3}$ do not represent only the anteroseptal regions. Therefore more sophisticated classification of anterior STEMI had been suggested. ${ }^{14}$

Zafrir et al. ${ }^{14}$ performed 17 segment based myocardial perfusion scan to see left ventricular segmental involvement in 55 patients with ASTEMI. The study showed involvement of basal segments occurring predominantly in AS-STEMI patients. On the contrary, mid and apical segmental dysfunction was more in EA-STEMI. This finding suggested more proximal occlusion of LAD in ASSTEMI than in EA-STEMI. No relation was observed regarding more lateral involvement with the presence of STE in leads I, aVL.

Engelen et al. ${ }^{11}$ also studied 100 patients of acute anterior STEMI to find out the difference of occlusion site in AS-STEMI and EA-STEMI and found no difference in occlusion site between the groups. This finding also implied that AS-STEMI is not a MI involving areas less than EA-STEMI.

Conclusion: The present study concludes that RWMA and global LV dysfunction in ECG pattern of acute AS-STEMI is not less extensive than that in the patients with the ECG pattern of acute EASTEMI. The term AS-STEMI neither implies that the ischemic process is limited to the anteroseptal segments, nor that the size of the ischemic area at risk is smaller than that in patients with EA-STEMI and it is as extensive as EA-STEMI. Both are equally devastating for patients. Therefore, the terminology "anteroseptal" is probably a misnomer and should not downgrade the extent of myocardial involvement as compared to that in EA-STEMI.
So, any patients with anterior wall involvement, either AS-STEMI or EA-STEMI, should be treated with equal importance.

\section{Acknowledgement}

We are thankful to the Department of Paediatric Surgery, Institute of Child and Mother Health for the financial support to conduct this study.

\section{References}

1. Kim MC, Kini AS, Fuster V. Definitions of Acute Coronary Syndromes. In: Fuster V, Walsh R, Harrington R. editors. Hurst's The Heart. $13^{\text {th }}$ ed. McGraw-Hill Medical Publisher, China; 2011. Chapter 56, p 1291,1293.

2. Rationale and design of the GRACE (Global Registry of Acute Coronary Events) Project: a multinational registry of patients hospitalized with acute coronary syndromes. Am Heart J. 2001; 141(2): 190-9.

3. Leo Schamroth. An Introduction to Electrocardiography. $7^{\text {th }}$ ed. Blackwell science Publisher Ltd; 1990. P 15,17,132,136.

4. Bandeali SJ, Stone S, Huang HD,Kayani WT, Wilson JM, Birnbaum Y. Comparison of segmental wall motion abnormalities on echocardiography in patients with anteroseptal versus extensive anterior wall STsegment elevation myocardial infarction. J Electrocardiol. 2012; 45: 551-55.

5. Shalev Y, Fogelman R, Oettinger M, Caspi A. Does the electrocardiographic pattern of "anteroseptal" myocardial infarction correlate with the anatomic location of myocardial injury? Am J Cardiol. 1995;75: 763-66.

6. Porter A, Wyshelesky A, Strasberg B, Vaturi M, Solodky A, Imbar $\mathrm{S}$ et al. Correlation between the admission electrocardiogram and regional wall motion abnormalities as detected by echocardiography in anterior acute myocardial infarction. Cardiology. 2000; 94: $118-126$.

7. Bogaty P, Boyer L, Rousseau L, Arsenault M. Is anteroseptal myocardial infarction an appropriate term? Am J Med. 2002; 113: 37- 41

8. Aldrich HR, Wagner NB, Boswick J,Corsa AT, Jones MG, Grande P, et al. Use of initial ST-segment deviation for prediction of final electrocardiographic size of acute myocardial infarcts. Am J Cardiol. 1988; 61: 749-53.

9. Huang HD, Tran V, Jneid H, Wilson JM, Birnbaum Y. Comparison of angiographic findings in patients with acute anteroseptal versus anterior wall ST-elevation myocardial infarction. Am J Cardiol. 2011; 107: 827- 32 .

10. Zhong-qun Z, Wei W, Jun-feng W. Does left anterior descending coronary artery acute occlusion proximal to the first septal perforator counteract ST elevation in leads V5 and V6? J Electrocardiol. 2009;42: 52-57. 
11. Engelen DJ, Gorgels AP, Cheriex EC, Muinck EDD, Ophuis AJO, Dassen WR, et al. Value of the electrocardiogram in localizing the occlusion site in the left anterior descending coronary artery in acute anterior myocardial infarction. J Am Coll Cardiol. 1999; 34: 389-95.

12. ACCF/AHA Guideline for the Management of STElevation Myocardial Infarction. 2013. Practice guideline. A Report of the American College of Cardiology. Foundation/American Heart Association Task Force on Practice Guidelines. Available from J Am Coll Cardiol.2013; 61(4): 78-140.

13. Cerqueira MD, Weissman NJ, Dilsizian V, Jacobs AK, Kaul S, Laskey WK, et al. Standardized myocardial segmentation and nomenclature for tomographic imaging of the heart: a statement for healthcare professionals from the Cardiac Imaging Committee of the Council on Clinical Cardiology of the American Heart Association. Circulation. 2002;105: 539-42.

14. Zafrir B, Zafrir N, Ben Gal T, Adler Y, Iakobishvili Z, Rahman MA et al. Correlation between ST elevation and $\mathrm{Q}$ waves on the predischarge electrocardiogram and the extent and location of MIBI perfusion defects in anterior myocardial infarction. ANE.2004; 9(2): 101-12.

15. Birnbaum Y, Saeed M, Ware D. Correlation Between the Electrocardiogram and Regional Wall Motion Abnormalities as Detected by Echocardiography in Patient with Acute Anterior and Inferior Myocardial Infarction. Int J Bioelectromagn. 2003; 5(1): 1- 3. 